Voix et Images

volxetimages

\title{
Gaston Miron : À bout portant
}

\section{Laure Hesbois}

Volume 4, numéro 1, septembre 1978

Rina Lasnier

URI : https://id.erudit.org/iderudit/200135ar

DOI : https://doi.org/10.7202/200135ar

Aller au sommaire du numéro

Éditeur(s)

Les Presses de l'Université du Québec

\section{ISSN}

0318-9201 (imprimé)

1705-933X (numérique)

Découvrir la revue

\section{Citer cet article}

Hesbois, L. (1978). Gaston Miron : À bout portant. Voix et Images, 4(1), 39-49.

https://doi.org/10.7202/200135ar d'utilisation que vous pouvez consulter en ligne.

https://apropos.erudit.org/fr/usagers/politique-dutilisation/ 


\section{Gaston Miron : À bout portant}

Miron appartient à cette génération de poètes québécois qui ont exprimé l'aliénation de leur peuple, et en l'exprimant, ont participé à l'émergence d'une conscience nationale, résumée dans la signification politique désormais affectée à l'adjectif "québécois». II est un de ces poètes pour qui création poétique et engagement politique ne font qu'un, l'une et l'autre étant la manifestation d'une situation existentielle intolérable.

Dans le contexte politique de 1953, date de la création de L'Hexagone, le francophone canadien est, selon l'expression même de Miron, un être "carencé ${ }^{1}$ ". Non seulement en tant que vaincu, dépossédé d'une terre que ses ancêtres ont conquise ou comme membre d'un groupe minoritaire défavorisé sur le plan économique et social mais, plus gravement, un homme agressé au plus profond de son être, un homme atteint dans sa culture, menacé dans sa langue, et par là même rendu étranger à lui-même et au monde, incapable d'accéder à l'identité et d'avoir une prise sur le réel. Par héritage tragique et par le décret d'autrui, il est le damned Canuck c'est-à-dire :

le sous-homme, la grimace souffrante du cro-magnon, l'homme du cheap way, l'homme du cheap work ${ }^{2}$,

sous-homme à qui il ne reste même pas la ressource d'élever sa protestation dans un langage articulé qui lui appartienne.

En proie à «la perversion sémantique à l'échelle de toute une langue $^{3}$, réduit au regard de l'altérité anglo-canadienne ${ }^{4}$, le poète n'en est pas moins tenu de témoigner: "Je n'ai que mon cri existentiel pour m'assumer solidaire de l'expérience d'une situation d'infériorisation collecti$v e^{5}$, dit Miron.

Gaston Miron, poète "empêché " ", c'est-à-dire poète dépossédé de ses signes, s'est donné pour mission première de «remettre la langue en fonctionnement ${ }^{7} \mathrm{~m}$. 
Cette situation est trop connue pour qu'il soit besoin d'y insister. Précisons, toutefois, que Miron en fait une analyse rigoureuse dans la dernière section de l'Homme rapaille, "Recours didactique", et plus particulièrement dans les Notes sur le non-poème et le poème. Sa position se trouve confirmée et précisée dans le texte d'une interview réalisée en 1973 par un groupe de l'Université Laurentienne ${ }^{B}$ et qui constitue une des analyses les plus pénétrantes du problème francophone que je connaisse.

II est clair que, pour Miron, la dépossession linguistique est la conséquence directe d'une aliénation politique et qu'il est vain d'espérer y remédier sans une révolution. Mais il est non moins évident que la vraie révolution d'un langage libéré, c'est la tension dialectique entre le poème et le non-poème:

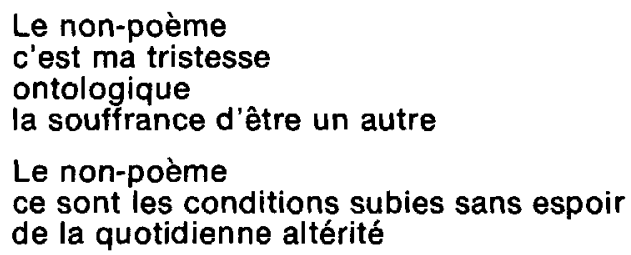

Or le poème ne peut se faire que contre le non-poème ne peut se faire qu'en dehors du non-poème car le poème est émergence car le poème est transcendance dans l'homogénéité d'un peuple qui libère sa durée inerte tenue emmurée

La racine du mal réside dans une pratique langagière tarée que Miron appelle la «diglossie». Non pas le bilinguisme qui maintient les deux codes relativement séparés, même quand on les utilise conjointement dans une même phrase, du type "le dispatcher m'a donné ma slip [pour "coupon"] pour aller gaser [faire le plein]", mais une confusion beaucoup plus subtile et plus dangereuse, parce qu'elle aboutit à une perversion radicale du signe, qui consiste à couler par insidieuse osmose des signifiés anglais dans des signifiants français. Prenons un exemple: "Voiture avec monnaie exacte seulement", lit-on sur l'autoroute des Laurentides. C'est un piège, dit Miron. En apparence c'est un texte clair: tous les signifiants sont français. Mais quel est le signifié de «monnaie exacte"? S'il existait, en fonction du code du français, 
ce serait quelque chose comme "argent de bon aloi», le contraire de "fausse monnaie" mais, de toute évidence, une telle interprétation ne s'adapte pas à la situation. En fait c'est "with exact change " qu'il faut comprendre. Et l'automobiliste, rompu à cette distorsion, jette docilement sa pièce de 25 cents dans le panier ${ }^{10}$.

Autre exemple: ascenseur «en usage». Faut-il comprendre qu'il pourrait être hors d'usage? Qu'il a été en panne, mais que par chance il vient tout juste d'être réparé et est à nouveau en état de fonctionner? Trève d'hypothèses gratuites. Chacun sait fort bien que l'appareil est tout simplement "in use" et attend patiemment que l'appareil occupé devienne libre. Une fois de plus l'utilisateur a opéré le rétablissement qui s'impose, mais à quel prix? Au prix d'une gymnastique intellectuelle qui tient de la schizophrénie. Au prix surtout d'une confusion des deux codes qui scelle son aliénation. En effet, dans cette sorte de symbiose, le français a perdu tout pouvoir de signifier directement. Le francophone n'a accès au réel que par le truchement d'une langue étrangère, ce qui, dans les propres termes de Miron, est la pire des dépossessions.

Je parle de ce qui me regarde, le langage, ma fonction sociale comme poète, à partir d'un code commun à un peuple. Je dis que la langue est le fondement même de l'existence d'un peuple, parce qu'elle réfléchit la totalité de sa culture en signe, en signifié, en signifiance. Je dis que je suis atteint dans mon âme, mon être, je dis que l'altérité pèse sur nous comme un glacier qui fond sur nous, qui nous déstructure, nous englue, nous dilue. Je dis que cette atteinte est la dernière phase d'une dépossession de soi comme être, ce qui suppose qu'elle a été précédée par l'aliénation du politique et de l'économique. Accepter $\mathrm{CECl}$ c'est me rendre complice de l'aliénation de mon âme de peuple, de sa disparition en l'altérité. Je dis que la disparition d'un peuple est un crime contre l'humanité car c'est priver celle-ci d'une manifestation différenciée d'elle-même. Je dis que personne n'a le droit d'entraver la libération d'un peuple qui a pris conscience de lui-même et de son historicité ${ }^{11}$.

D'autre part, faute d'un langage qui lui soit propre, il n'a aucun moyen d'exprimer lui-même ses déterminations, sa spécificité, et n'a le choix qu'entre l'insignifiance d'une expérience individuelle incommunicable ou la dépersonnalisation qui résulte du recours aux catégories universelles. Coupé du monde extérieur, il est de surcroît condamné à l'autisme ou à la désintégration. A moins qu'il ne préfère une solution plus radicale encore et n'opte pour l'assimilation pure et simple. Dans tous les cas, son identité lui échappe et c'est finalement l'autre qui en décide :

Longtemps je n'ai su mon nom, et qui j'étais, que de l'extérieur. Mon nom est "Pea Soup". Mon nom est "Pepsi". Mon nom est "Marmelade». Mon nom est "Frog". Mon nom est "dam Canuck». Mon nom est «speak white». Mon nom est "dish washer». Mon nom est «floor sweeper». Mon nom est «bastard». Mon nom est « cheap». Mon nom est "sheep". Mon nom... Mon nom... ${ }^{12}$ 
Passe encore si l'anglais ne faisait qu'imposer son lexique! Mais, comme on le voit par l'exemple précité, la structure même est atteinte. L'affirmation de soi se moule dans une forme empruntée : «mon nom est..."

II y a donc, à l'origine, une pollution du langage et, à partir de là, une occultation du réel et une dégradation de l'être qui va jusqu'à l'anéantissement. C'est de cette déréliction absolue, traduite par Miron dans une série de termes à préfixe négatifs - dé-réalisation, dé-création, décentré, déshumanisé, déphasé, déculturé, dépoétisé - que naît le poème.

Moi je gis, muré dans la boîte crânienne

dépoétisé dans ma langue et mon appartenance

déphasé et décentré dans ma coïncidence

ravageur je fouille ma mémoire et mes chairs

jusqu'en les maladies de la tourbe et de l'être

pour trouver la trace de mes signes arrachés emportés

pour reconnaître mon cri dans l'opacité du réel ${ }^{13}$

La poésie de Miron est une réaction d'autodéfense, il l'avoue.

Je défends ma peau
rien que ça
ma peau de peau
c'est bien assez
il me semble
pour commencer ${ }^{14}$

C'est son ultime recours contre la malédiction qui pèse sur lui. Prendre la parole, c'est-à-dire reprendre possession de ses signes, est le seul moyen de rendre au vécu sa signification et à l'homme sa dignité.

L'affirmation de soi, dans la lutte du poème, est la réponse à la situation qui dissocie, qui sépare le dehors et le dedans ${ }^{15}$.

Le poème se fait contre et malgré le non-poème. Et le poème refait l'homme. Mais l'entreprise n'est pas facile:

Comment dire ce qui ne peut se confier? Je n'ai que mon cri existentiel pour m'assumer solidaire de l'expérience d'une situation d'infériorisation collective. Comment dire l'aliénation, cette situation incommunicable? Comment être moi-même si j'ai le sentiment d'être étranger dans mon objectivité, si celle-ci m'apparaît comme opaque et hostile, et si je n'existe qu'en ma subjectivité ${ }^{16}$ ?

Comment rétablir la communication, à partir d'un langage vicié?

Le plus simple est sans doute de prendre appui sur quelques signes privilégiés, ceux qui continuent à fonctionner à peu près normalement et à travers lesquels une collectivité peut se reconnaître. J'en citerai trois catégories:

$1^{\circ}$ certains termes concrets se réfèrent à une réalité spécifique et qui fonctionnent plus ou moins comme signes de ralliement: achigan, fardoche, ouananiche, bois-debout, etc. 
$2^{\circ}$ certains termes dotés d'un coefficient affectif marqué, onomatopées, interjections, qui trouvent un écho naturel dans une situation déterminée. Au premier rang de ceux-là, il convient de placer les sacres, expression spontanée d'une impatience et d'une révolte :

Sainte Bénite de Sainte Bénite de batèche

Sainte Bénite de vie magannée

belle grégousse de vieille réguine de batèche ${ }^{17}$

$3^{\circ}$ une série de termes ou d'expressions idiolectales, classées comme canadianismes dans tout bon dictionnaire, en tout cas suffisamment différenciées et suffisamment chargées de connotations culturelles pour créer une solidarité : maganer, garrocher, désâmer, amanchures, parlures, sacrures, gagnages, etc. Quand Miron chante :

Mon accotée

ma tannante de belle accotée ${ }^{18}$.

il est clair qu'il se situe géographiquement et socio-culturellement. Et nul "étranger" ne parviendra jamais à lire avec l'accent convenable ces extraits de “la Batèche».

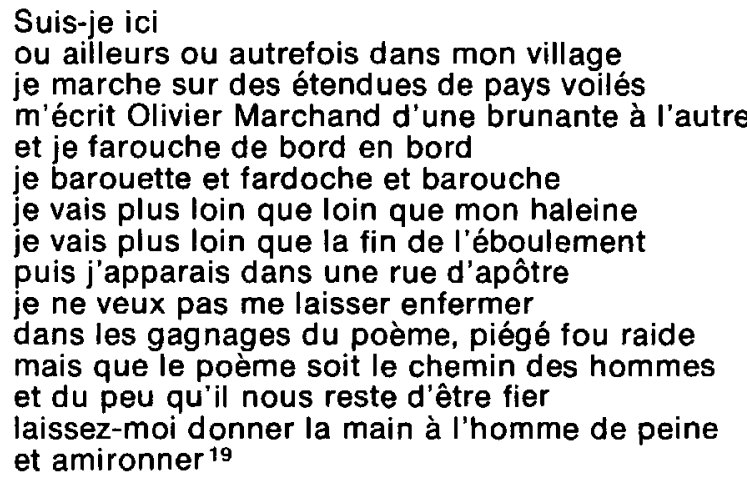

Cré bataclan des misères batèche cré maudit raque de destine batèche raque des amanchures des parlures et des sacrures moi le raqué de partout bateche

nous les raqués de l'histoire batèche ${ }^{20}$

Comme on peut en juger, Miron ne recule pas devant les termes à saveur locale. Mais il se garde bien de tomber dans un régionalisme de complaisance. II sait trop bien que le pittoresque, pour un peuple, n'est le plus souvent qu'une forme insidieuse de son infériorisation: "C'est l'autre qui qualifie ainsi ma différence et ce faisant, me réduit à ma seule apparence extérieure, que de surcroît il minimise." II se méfie à juste titre de la folklorisation qui équivaut, en fait, à une extermination sans douleur ${ }^{21}$. Par conséquent, s'il parle de “boucane», de «gigue", de "peau de babiche" et autres accessoires de la panoplie folklo- 
rique, c'est simplement parce que ces termes renvoient à un vécu privilégié, évoquent des vestiges culturels auxquels l'anglais n'a pas de part et échappent par là même à la pollution du bilinguisme. Parce que ce sont des termes qui fonctionnent encore de façon à peu près normale, et qu'il faut bien commencer avec ce que l'on a :

J'avance en poésie, dit Miron, avec les maigres mots frileux de nos héritages avec la pauvreté natale de ma pensée rocheuse 22

Mais ce n'est que le point de départ d'une ouverture qui vaut d'être notée. Chez Miron le trait spécifique est un moyen de rejoindre l'universel :

Tu es mon amour, ma ceinture fléchée d'univers ma danse carrée des quatre coins d'horizon ${ }^{23}$

Compte tenu de la situation où il se trouve et des motifs qui le poussent à prendre la parole, on ne s'étonnera pas non plus de voir Miron créer de nouveaux termes pour suppléer aux déficiences éventuelles d'un langage mutilé. Le “québécanthrope", ainsi qu'il le nomme, est en effet une race d'homme à part. Une race de déshérités rebelles ${ }^{24}$. La langue dont il dispose est, par définition, une langue appauvrie, polluée. Mais, telle quelle, elle demeure son seul recours contre l'assimilation. II se trouve donc contraint de préserver ce qui en reste et, au besoin, de la réinventer. Dans cet effort, archaïsmes et néologismes se mêlent à plaisir. En effet, qu'il s'agisse de formes vieillies, conservées en hommage à ses origines, ou de créations nouvelles, attestant sa survie, il s'agit avant tout de combler les vides, de parer aux déviations. Miron le fait avec bonheur: la “désespérance" est un mal plus ancré, plus chronique que le désespoir. Les “partances" sont plus déchirantes que de simples départs. La «brunante " décrit joliment la tombée de la nuit et a, sur crépuscule, la supériorité d'être simple. Bien qu'il se conforme le plus souvent à des modèles éprouvés, Miron ne s'embarrasse pas, le cas échéant, des règles morphologiques. Il innove en fonction du besoin immédiat. C'est ainsi qu'à côté de formations régulières comme «buissonneuse", [nuit] "faillible", [mon courage] "pagayeur" [mes bras], il n'hésite pas à proposer, «médiantes» [eaux]. On peut contester la rigueur du procédé, on ne saurait nier l'expressivité de ses trouvailles: «je me désoleille, je rafale, je charbonne", déclare-t-il au hasard des pages, ou encore: "mon corps t'enhoule, cargue moi en toi », et en guise d'art poétique :

ma pauvre poésie tel un amour chez les humbles de perce-neige malgré les malheurs de chacun de perce-confusion de perce-aberration ${ }^{25}$...

Accordons-lui licence de lutter avec les moyens dont il dispose, et s'il le faut, d' «amironner" pourvu que le poème reste ainsi qu'il le dit "le chemin des hommes ${ }^{26}$ ". 
A ces expressions neuves, forgées pour les besoins de la cause, il faut ajouter l'usage surprenant que Miron fait parfois des termes courants. Maladresse congénitale? Miron l'affirme : «Je suis un homme simple avec des mots qui peinent... ${ }^{27}$ " ou mieux encore :

Celui qui n'a rien comme moi, comme plusieurs marche depuis sa naissance, marche à l'errance avec tout ce qui déraille et tout ce qui déboussole dans son vague cerveau que l'agression embrume ${ }^{28}$

Mais n'est-ce pas plutôt le gauchissement imposé à qui se sert d'un outil faussé? Les formes de la prose quotidienne ne peuvent dire que l'aliénation. Celles qu'on emprunte au français standard sont inadéquates à la situation, donc le poète est condamné à faire du "sur mesure". Certes, toute parole authentique suppose un ajustement du code et, plus que tout autre, la parole poétique peut être considérée comme un écart par rapport à la norme. Mais, pour Miron, le problème se trouve en quelque sorte inversé. La norme n'existe pas. II ne s'agit pas pour lui, d'exprimer l'originalité d'une expérience individuelle à partir du code commun mais de hausser une expression personnelle au niveau d'une expérience collective. II fait œuvre de pionnier. Et, ce faisant, il confère un sens nouveau à certains termes. L'adjectif "natal", entre autres, se charge chez lui de toutes les servitudes et de toutes les exigences biologiquement liées à l'origine: "mes chairs natales", dit-il s'adressant à sa mère; il dénonce le froid "natal " et, inversement, revendique «le soleil natal ", "une langue natale ", une terre "qui te soit natale".

Par une légère altération, il redonne vie à des expressions usées: “comme d'accoutumance - Je bêle à la mort - à travers nuits et gués au gré des avenirs mortes" -, etc.

Mais, surtout, il multiplie les sens possibles :

Ma poésie le cœur heurté

Ma poésie de cailloux chahutés ${ }^{29}$

écrit Miron, confondant le choc émotif et les battements du cœur, les secousses d'un chemin raboteux et la véhémence de sa protestation. Ou encore, ajoutant le poids de la honte à la rigidité du cadavre :

empesés d'humiliation et de mort

Est-ce l'image d'une épave ou l'échec d'une vie que Miron cherche à traduire dans: "le sourire échoué du pauvre avenir avili"? Qu'importe! $A$ qui «prend sur soi de ne pas mourir ${ }^{30}$ " et parle en jouant sa vie, on ne demande pas de consulter les manuels du bon langage. Pour Miron tout est bon qui peut faire sens : de l'usage local attesté par exemple dans "les brouillards de chagrin crus", "la peine débonde", à l'étymologie la plus stricte ("tu conjoins en ton vol la terre et l'espace ${ }^{31}$ ), en passant par toute la gamme des figures de rhétorique, accumulées à plaisir, calembour y compris. 
C'est dans le même esprit, me semble-t-il, qu'il faut interpréter les irrégularités syntaxiques dont il se rend coupable à tout bout de champ. Prenons quelques exemples:

1. Tout d'abord, le mélange des catégories, comme l'emploi d'un adjectif à la place d'un adverbe ou inversement : «regretter continu ", "saigner dur», "une conscience ensemble" ou anomalie plus frappante encore, d'un nom employé comme adjectif : "l'aïeule prière de nos doigts".

2. Ajoutons à cela, l'emploi des verbes transitifs sans complément d'objet et vice-versa : "la vie refoule", «mon angoisse poudrait», "la lune feuillette", «les balcons colportent», mais au contraire, s'adressant à sa poésie :

Ma pauvre poésie dans les nippes de famille

de quel front tu harangues tes frères humiliés

de quel droit tu vocifères ton sort avec eux ${ }^{32}$.

ou encore :

tu me hầtes en toi, c'est ma vie que j'ai mal, je m'écris sous la loi d'émeute, tes nœuds m'enchevêtrent.

3. II arrive aussi que, sans modifier la structure, Miron la fasse cependant éclater, par l'utilisation aberrante qu'il en fait: «j'ai eu noir, j'ai eu froid», se plaint-il, ou plus audacieusement encore : «Moi j'ai noir éclaté dans la tête».

4. Un bon nombre des structures nominales que l'auteur affectionne semblent en fait obtenues par transformation à partir d'une locution verbale à laquelle il s'efforce ainsi de donner plus de poids. C'est le cas lorsqu'il se présente comme «concasseur de désespoir», qu'il invoque ses poings "cogneurs de folles tendresses" ou, s'adressant au pays, jure de se faire «hâleur de son avènement". Notons au passage que les locutions verbales dont procèdent de telles expressions résultent le plus souvent d'une réinterprétation personnelle de lexies figées : "concasser du désespoir" est une réactivation énergique et heureuse du cliché «broyer du noir».

5. Signalons pour finir une curieuse formule de renforcement chère à Miron, qui consiste à répéter un nom, sous forme de faux complément: "ma tête de tête, ma peau de peau, mes yeux de z-yeux, ma vie de vie», etc.

Le résultat de ces diverses violations, ce sont des phrases gauchement bâties, "raboteuses", dirait Miron, mais qui du moins défient les pièges de la diglossie et les stérotypes du français officiel. Des phrases à la mesure exacte d'un homme parlant au nom de son peuple bâillonné, et qui, en l'absence d'un code sûr, s'applique à articuler, parfois un peu anarchiquement, des signes un à un reconquis. La désinvolture à l'égard des normes n'est évidemment pas un trait exclusif à Miron. 
Chacun sait que les pulsions vitales prises en charge par la poésie s'accommodent mal des rigueurs syntaxiques et que, d'une manière ou d'une autre, tout poète en arrive à violer le code. Mais, chez Miron, la parole, toute proche du cri existentiel, précède, historiquement, l'établissement du code communautaire. II est, pour ainsi dire, condamné à être poète.

Qui dit poésie dit révélation d'un monde inédit, ce qui ne peut se faire que par substitution analogique, autrement dit par métaphore. La poésie de Miron ne fait pas exception à la règle. Mais, ici encore, il importe de distinguer: le déplacement de sens qu'opère cette figure représente tantôt un raffinement d'expression supposant une parfaite maîtrise du cơde, et tantôt un expédient plus ou moins habile, trahissant la gêne. Chez Miron cela ressemble fort au trébuchement d'un homme talonné par le danger, qui n'a pas le temps de s'embarrasser de subtilités: brancarts ou harnais, c'est tout un pour le cheval rétif; dans un cas comme l'autre il s'agit d'une atteinte à sa liberté. Ruer ou se ruer contre, quelle différence cela peut-il faire, pourvu qu'on manifeste son refus? Et pour peu que le cri viscéral s'ajoute à l'évocation du cheval cabré pourquoi Miron n'écrirait-il pas : "Je me hurle dans mes harnais ${ }^{33}$ ? "

Peut-on demander à un être affolé de souffrance de distinguer entre deux modes de torture, entre les coups de pioche et les coups d'épieu? Et en effet Miron mélange tout: "Je suis pioché d'un mal d'épieu ${ }^{34}$."

Tout se passe comme si, entre deux représentations possibles, l'auteur refusait de faire un choix. Ainsi s'expliquent notamment ces signes composites, ces couples de termes si caractéristiques du style de Miron, que ce soit sous forme d'une apposition directe ou d'un pseudocomplément : "l'espace rouge-gorge", «nos rêves bourrasques ", "l'amour tocsin», «mon cceur derrick», «mon cœur obus», "l'accord comète de tes plaines", "ton corps de voie lactée», etc. L'emploi du terme propre n'exclut pas, pour Miron, l'adjonction d'un terme imagé, simplement accolé au précédent: «un souvenir de bulle», «un matin d'obus lilas", “la mort acétylène", «ma tête grenade et déflagration». Gardons-nous bien, ici encore, d'interpréter comme un trait individuel, une figure inhérente à la poésie en général. Il est devenu banal, depuis Hugo, de conjoindre dans une même locution deux aspects différents de la réalité (exemple canonique: le pâtre-promontoire) et les surréalistes se sont presque fait un dogme de ce type de courts-circuits, qu'ils considéraient comme le plus sûr moyen de rompre avec nos habitudes mentales. Contentons-nous de noter que cette façon de s'exprimer convient particulièrement bien à qui trébuche à la recherche de ses signes, à qui procède

par contiguïté, par conglomérat par mottons de mots ${ }^{35}$

à qui, pour tout dire, la simple prose est impossible! 
La meilleure définition du style de Miron, c'est finalement lui-même qui nous la propose, dans un poème intitulé : «A bout portant». Ce texte a d'abord paru dans la revue Parti pris en 1968 et a été repris en 1975 dans une publication de l'Université d'Ottawa, dans une version antérieure semble-t-il, puisqu'il est daté de 1958.

\title{
A BOUT PORTANT
}

\begin{abstract}
A bout portant à tout bout d'champ pas de temps pour le beau mot pas de temps pour l'extase le scintillement le tour noble ces jeux qui ourleraient si bien la poésie

pas de temps le temps est au plus mal la vie va vite la vie à chaux la vie à vau et la mort est vaste la mort en tas menace

pas de temps pour le temps le temps nous manque faut ce qu'il faut tirer juste et juste à temps à bout portant à tout bout d'champ
\end{abstract}

\section{(La Vie agonique ${ }^{36}$ )}

De fait, toute la poésie de Miron est une sorte de cri, jailli d'une urgence existentielle, et qui cherche sa forme. On connaît la difficulté d'éditer cet auteur : c'est que ses poèmes ne sont pas des textes, des écrits composés en vue d'une certaine durée, mais bel et bien des paroles transitoires, hâtivement notées sur des bouts de papier qu'il s'obstine à perdre. Chaque version présente des variantes par rapport à la précédente.

Le poème que je viens de citer constitue lui-même un échantillon exemplaire des traits que j'ai tenté de dégager.

A bout portant, à tout bout d'champ.

Pour commencer, à la faveur d'une similitude fortuite entre les signifiants, Miron fond deux expressions exprimant respectivement la proximité du danger et la routine, faisant donc d'une situation critique un état chronique. Dans ces conditions, parler n'est pas un exercice de virtuosité. C'est la seule parade possible contre une menace mortelle :

«Pas de temps pour le beau mot», dit Miron. Si l'on compare les deux versions, ce caractère d'urgence devient encore plus sensible. Dans le texte d'Ottawa on a : «la vie va vite, à chaud, à vau l'eau, en queue dveau», chaud écrit c.h.a.u.d comme dans : opérer à chaud, c'est-àdire en état de crise aiguë. Dans le texte de Parti pris, cela devient la vie à chaux, c.h.a.u.x. c'est-à-dire un matériau de construction éminemment friable et sans cesse exposé à la désintégration. L'agression est constante: la riposte doit être immédiate. II ne s'agit pas de faire le délicat. On fait avec flèche de tout bois.

Faut ce qu'il faut, tirer juste et juste à temps

A bout portant, à tout bout d'champ. 
Je suis toute disposée, pour ma part, à lui accorder un brevet de tireur d'élite.

\author{
Laure Hesbois \\ Université Laurentienne
}

\begin{abstract}
A moins d'indications contraires, toutes les citations sont extraites de l'Homme rapaillé, Montréal, Les Presses de I'Université de Montréal, 1970. On se bornera donc à indiquer le titre du poème et la page.
\end{abstract}

1. «Un long chemin", p. 121.

2. "Le Damned Canuck», p. 55.

3. "Notes sur le non-poème et le poème", p. 128.

4. "Un long chemin ", p. 116.

5. "Notes sur le non-poème et le poème ", p. 128.

6. Ibid., p. 127.

7. Bande enregistrée d'une interview réalisée par Robert Dickson le 31 mars 1973.

8. Un extrait de cette rencontre a été publiée dans la Revue de l'Université Laurentienne, vol. IV, $n^{\circ} 2$, p. 11-17.

9. "Notes sur le non-poème et le poème", p. 122-123.

10. Interview du 31 mars 1973.

11. "Notes sur le non-poème et le poème ", p. 124.

12. Ibid., p. 127.

13. "Monologue de l'aliénation délirante ", p. 58-59.

14. "Self-defence", p. 25.

15. «Notes sur le non-poème et le poème $*$, p. 128.

16. Ibid.

17. Courtepointes, Ottawa, Éditions de I'Université d'Ottawa, 1975.

18. Séquences de "la Batèche», p. 44-45.

19. Courtepointes, p. 39.

20. Séquences de «la Batèche», p. 44-45.

21. Voir en particulier, «Un long chemin», p. 121.

22. «Dans les lointains $\approx$, p. 77.

23. "La Marche à l'amour", p. 38.

24. Voir p. 45 "Je suis un homme simple avec des mots qui peinent..."

25. "La Pauvreté anthropos ", p. 76.

26. Courtepointes. Séquences de «la Batèche», p. 44.

27. "Avec toi ", p. 45.

28. "Les Années de déréliction ", p. 81.

29. "Ma désolée sereine », p. 23.

30. Courtepointes, p. 31.

31. "La Corneille", p. 78.

32. "La Pauvreté anthropos», p. 76.

33. "Notes sur le non-poème et le poème", p. 123.

34. "Poème de séparation $1 \%$, p. 42.

35. "Notes sur le non-poème et le poème", p. 127.

36. Version de Parti pris, vol. $V, n^{\circ} 8, p .78$. 